09.2;05.1

\title{
Использование спекловых изображений для определения локальных пластических деформаций, возникающих при многоцикловой усталости стали 09Г2C
}

\author{
(C) А.П. Владимиров ${ }^{1,2}$, Н.А. Друкаренко ${ }^{1}$, К.Е. Мызнов ${ }^{2}$ \\ ${ }^{1}$ Институт машиноведения УрО РАН, Екатеринбург, Россия \\ ${ }^{2}$ Уральский фредеральный университет им. Б.Н. Ельцина, Екатеринбург, Россия \\ E-mail: vap52@bk.ru
}

Поступило в Редакцию 4 марта 2021 г.

В окончательной редакции 16 апреля 2021 г.

Принято к публикации 4 мая 2021г.

С использованием усредненных во времени спекловых изображений на образце из стали 09Г2С, изготовленном с двумя выточкам радиусом $2.5 \mathrm{~mm}$, оценены пластические деформации, возникающие в зоне зарождения усталостной трещины. Показано, что усталостное разрушение возникает вследствие локализации необратимых процессов в области размером менее $1 \mathrm{~mm}$, предельное значение растягивающих пластических деформаций имеет порядок $10^{-1}$. В качестве предвестника разрушения предложено использовать уменьшение нормированной временно́й автокорреляционной функции интенсивности излучения до отрицательного значения.

Ключевые слова: спеклы, изображение, многоцикловая усталость, деформация, разрушение.

DOI: 10.21883/PJTF.2021.15.51232.18752

Актуальность изучения многоцикловой усталости связана с тем, что от 50 до 70\% деталей техники разрушаются вследствие этого типа усталости [1-3]. Несмотря на большую историю исследований [4-6] и многочисленные публикации $[7,8]$, в настоящее время отсутствуют методы неразрушающего контроля и оценки остаточного ресурса деталей, работающих в условиях многоцикловой усталости, которые удовлетворяли бы требованиям инженерной практики [2]. Сразу после создания лазеров и обнаружения спекловой структуры рассеянного излучения спеклограммы и голограммы были использованы для изучения усталостных явлений [9-11], однако из-за немонотонного изменения регистрируемых сигналов и трудоемкости методик они не получили широкого распространения. Указанные выше недостатки спекловых и голографических методов были преодолены в [12-14], а в работе [13] было дано теоретическое обоснование метода усредненных во времени спекловых изображений (спекл-картин в плоскости изображения объекта), продемонстрировано его использование для количественного определения необратимых деформаций, возникающих при испытании стали на многоцикловую усталость, оценены погрешности измерений. Метод позволяет на базе порядка $10 \mu \mathrm{m}$ надежно определять относительные перемещения центров рассеяния поверхности на величину порядка $10 \mathrm{~nm}$. В работе [14] показано, что метод по чувствительности и пространственному разрешению на один-два порядка превосходит имеющиеся традиционные методы корреляционной и голографической интерферометрии. В указанных выше работах [12-14] оптическая система позволяла определять проекцию вектора относительного перемещения точек поверхности на ее нормаль. Кроме того, для локализации необратимых процессов на заранее известном месте были использованы образцы с острым надрезом типа Шарпи, с радиусом закругления на вершине надреза, равным $0.25 \mathrm{~mm}$. Было показано, что в процессе зарождения трещины на вершине надреза возникает зона пластических деформаций размером менее $1 \mathrm{~mm}$. В настоящей работе использовались образцы со слабым концентратором напряжений, а именно с двумя симметрично расположенными выточками с радиусами $2.5 \mathrm{~mm}$. В ходе испытания образца на усталость впервые на малой базе порядка $10 \mu \mathrm{m}$ были определены изменения не одной, а трех компонент указанного вектора. Основной целью работы было определение предельных значений трех компонент вектора относительного перемещения точек поверхности. Отметим, что для изучения особенностей пластических деформаций можно использовать различные традиционные оптические [15-17] и неоптические [1,18-20] методы. Однако при их использовании для измерений на малых базах возникают большие методические трудности.

Ранее на основе модели отражающего объекта в виде совокупности точечных центров рассеяния, расположенных на ее поверхности, в работе [13] была решена задача о динамике спеклов в плоскости изображения периодически деформируемого объекта, были получены формулы для интенсивности излучения $\tilde{I}$ в некоторой точке плоскости изображения и нормированной временно́й автокорреляционной функции $\eta\left(t_{1}, t_{2}\right)$ этой интенсивности. Предполагалось, что величина $\tilde{I}$ является усредненной по времени интенсивностью излучения, а время усреднения равно или кратно периоду $T$ цикличе- 


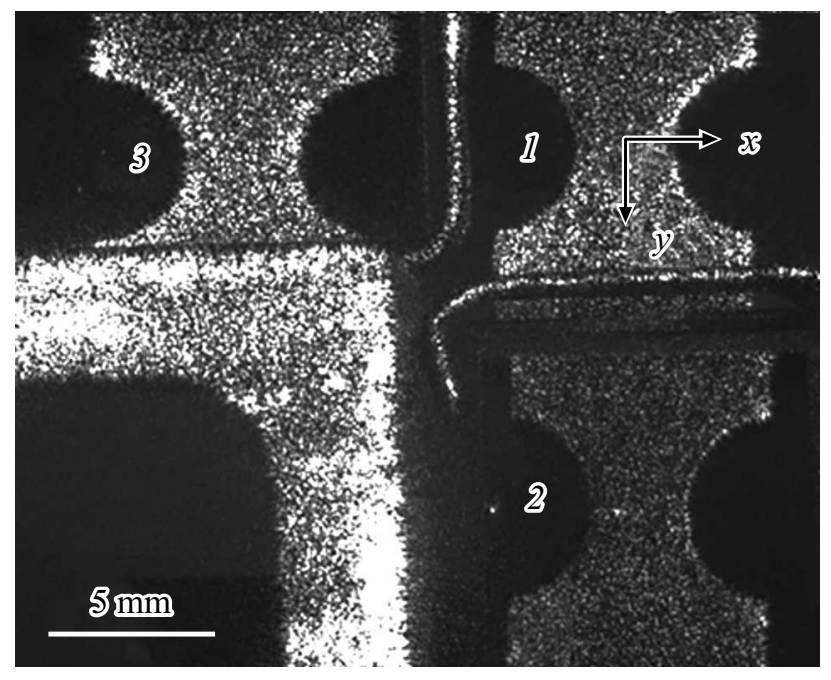

Рис. 1. Три спекловых изображения $(1-3)$ образца на одном кадре.

ских нагрузок. Для I I было получено

$$
\begin{gathered}
\tilde{I}=I_{1}+I_{2} \exp \left[-\sigma^{2} / 2\right] \cos [x+\alpha], \\
\eta\left(t_{1}, t_{2}\right)=\eta_{1} \eta_{2} \eta_{3}=\eta\left(u_{x}\right) \cos \left[\left\langle x_{2}\right\rangle-\left\langle x_{1}\right\rangle\right] \\
\times \exp \left[-\frac{1}{2} k_{11}-\frac{1}{2} k_{22}+k_{12}\right] .
\end{gathered}
$$

В формуле (1) $I_{1}, I_{2}, \alpha-$ константы, $x$ и $\sigma^{2}-$ среднее значение и дисперсия величины $k \Delta \mathbf{u}\left(\mathbf{I}_{s}+\mathbf{I}\right), k=2 \pi / \lambda-$ волновое число, $\Delta \mathbf{u}-$ вектор относительного перемещения двух центров рассеяния, расположенных в области, размер которой $\Delta y$ равен линейному разрешению линзы, $\mathbf{I}_{s}$ и I - единичные векторы, направленные от центра области к источнику света и к центру линзы соответственно. В (2) $\eta_{1}=\eta\left(u_{x}\right)$ - автокорреляционная функция, соответствующая поступательному перемещению объекта, где для определенности предполагалось, что объект перемещается по оси $o x,\left\langle x_{1}\right\rangle$ и $\left\langle x_{2}\right\rangle$ - средние значения, $k_{11}$ и $k_{22}$ - дисперсии, а $k_{12}$ - смешанный корреляционный момент величин $x$ в моменты времени $t_{1}$ и $t_{2}$ соответственно, угловые скобки означают усреднение по ансамблю объектов.

На рис. 1 приведен типичный кадр с тремя спекловыми изображениями плоской рабочей части образца толщиной $1.6 \mathrm{~mm}$, соответствующими трем ракурсам наблюдения. Изображение, обозначенное цифрой 1 , регистрировалось путем наблюдения по нормали поверхности. Изображения 2 и 3 формировались с использованием двух одинаковых призм, отклоняющих рассеянные объектом спекл-модулированные волны в сторону объектива телекамеры. Параметр шероховатости поверхности $R_{a}$, найденный с помощью интерференционного микроскопа WYKO NT-1100, был равен $0.8 \mu \mathrm{m}$. До и после испытания образца на усталость для обнаружения трещин прибором WYKO NT-1100 регистрировались трехмерные профили поверхности. Циклическое нагружение образцов проводилось на высокочастотной резонансной испытательной машине „MIKROTRON“ Rumul по схеме отнулевого растяжения при частоте нагружения $\sim 100 \mathrm{~Hz}$ и двойной амплитуде $2.0 \mathrm{kN}$. Образец освещался лазерным модулем с длиной волны $\lambda=0.65 \mu \mathrm{m}$ мощностью $20 \mathrm{~mW}$. В эксперименте была использована монохромная телекамера типа ВИДЕОСКАН-415M-USB с матрицей, содержащей $782 \times 582$ фотоэлемента размером $8.3 \times 8.3 \mu \mathrm{m}$. Время усреднения $0.5 \mathrm{~s}$ соответствовало 53 циклам нагружения. Размер диафрагмы объектива телекамеры подбирался таким, чтобы минимальный размер спеклов был несколько больше размера фотоэлемента матрицы фотоприемников телекамеры. Значение $\eta$ на фрагменте изображения размером в $m \times m$ пикселей определялось по формуле (38) из работы [13]. Оригинальное программное обеспечение текущие кадры (т.е. через 53 цикла деформации), а также кадры через каждые 1000 циклов нагружения записывало в отдельные файлы для их обработки после проведения опыта. Испытание образцов прекращалось при изменении резонансной частоты на $10 \%$.

На рис. 2 приведены распределения величины $\eta$ вдоль вертикальной линии на изображении 2 образца, размеры фрагмента изображения $5 \times 5$ пикселей. Линия проходила через область спеклового изображения, которая соответствовала участку поверхности вблизи выреза, где в конце опыта зародилась трещинка длиной порядка $100 \mu \mathrm{m}$. Как видно из приведенных кривых, необратимые процессы протекают неоднородно, имеются локальные зоны с повышенным значением пластической деформации. Начиная примерно с 30000 циклов в одной из таких зон начались необратимые процессы, уменьшающие величину $\eta$ до отрицательных значений. Именно в этой зоне и была обнаружена трещина. Построение

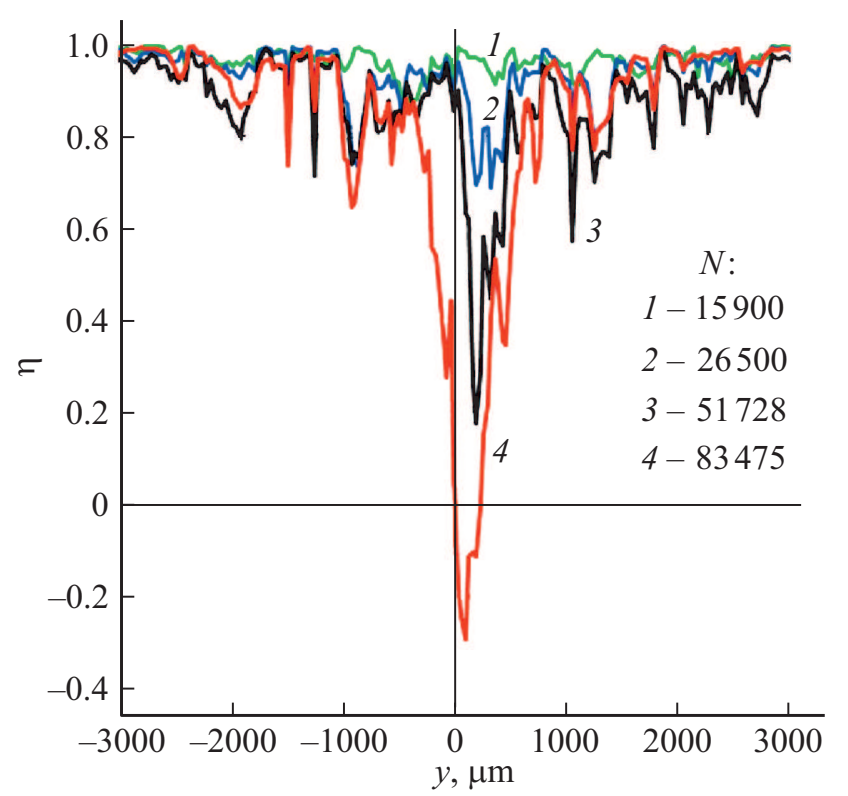

Рис. 2. Зависимости $\eta(y)$ для разных циклов $N(x \cong 3.0 \mathrm{~mm})$. 

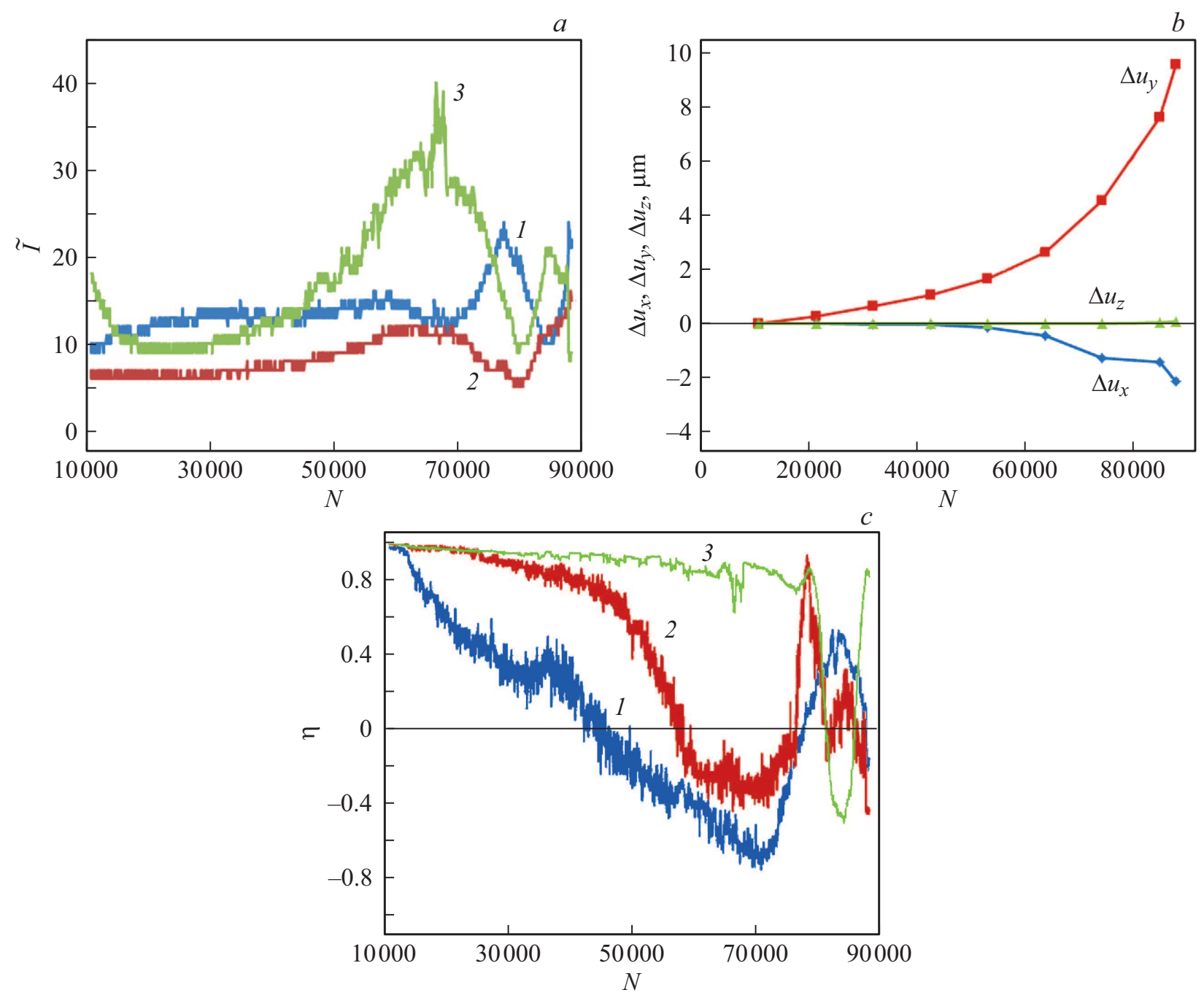

Рис. 3. $a$ - зависимости $\tilde{I}(N)$ для трех ракурсов наблюдения (номера кривых соответствуют номерам изображений образца); $b$ - зависимости компонент вектора $\Delta \mathbf{u}$ от числа циклов $N$; $c$ - зависимости параметра $\eta$ от числа циклов $N$ (номера кривых соответствуют номерам изображений образца).

аналогичной зависимости $\eta(y)$ вдоль линии, проходящей через участок, соответствующий центру образца, показало, что минимальное значение величины $\eta$, равное 0.38 , достигается в одной из локализованных зон деформации. На рис. 3, $a$ для трех ракурсов наблюдения приведены зависимости цифрового значения интенсивности излучения $\tilde{I}$ от числа циклов нагружения $N$. Пиксели на трех изображениях соответствовали одной области зарождения трещины, а именно центру зоны локализованной деформации. Как видно из приведенных кривых, зависимости $\tilde{I}(N)$ являются квазипериодическими функциями. Согласно формуле (1), максимальным значениям $\tilde{I}$ соответствуют значения аргумента косинуса $x+\alpha$, равные $\pm 2 \pi n(n=1,2,3)$. Минимальным значениям кривых отвечают значения аргумента, равные $\pm \pi(1+2 n)$. Для трех кривых нами были построены зависимости $n$ от $N$, все величины были взяты со знаком ,плюс“. Экспериментальные зависимости $n(N)$ аппроксимировались полиномами третьей и четвертой степени, достоверность аппроксимации была не менее 0.998. Далее из всех значений $n$ вычитались их начальные значения $n_{0}$. Затем с шагом 10000 циклов по известным функциям определялись три значения величин $\Delta n=n-n_{0}$, соответствующие трем направлениям наблюдения. В результате решения трех уравнений $\Delta \mathbf{u}\left(\mathbf{I}_{s}+\mathbf{I}\right)=\lambda \Delta n$ с известными из опыта значениями компонент векторов $\mathbf{I}_{s}$, $\mathbf{I}$ для каждого выбранного цикла определялись три компоненты $\Delta u_{x}, \Delta u_{y}, \Delta u_{z}$ вектора $\Delta \mathbf{u}$. В уравнениях использовались единичный вектор $\mathbf{I}_{s}(0,-0.190,0.981)$, единичные векторы $\mathbf{I}(0,0,1), \mathbf{I}(0,0.310,0.951), \mathbf{I}(0.308,0,0.982)$, соответствующие изображениям 1,2 и 3 . При решении системы уравнений знак величины $\Delta n$ брался из физических соображений, а именно из условия достижения величиной $\Delta u_{z}$ минимального значения. На рис. $3, b$ приведены совмещенные зависимости величин $\Delta u_{x}, \Delta u_{y}, \Delta u_{z}$ от числа циклов $N$. Видно, что максимальное относительное необратимое перемещение точек поверхности, усредненное как по времени, так и по 
области размером $\Delta y=66 \mu \mathrm{m}$, приближается к $10 \mu \mathrm{m}$. Следовательно, предельная растягивающая пластическая деформация поверхности, оцененная нами как $\Delta u_{y} / \Delta y$, достигает величины порядка $10^{-1}$. Анализ зависимостей $\eta(y)$, найденных при разных значениях $N$, а также $\eta(N)$, соответствующих разным фрагментам изображения, показал, что в качестве предвестника разрушения элемента поверхности целесообразно использовать факт уменьшения величины $\eta$ до отрицательного значения. На рис. 3, $c$ для трех направлений наблюдения приведены зависимости $\eta(N)$, соответствующие зоне зарождения трещины, размер фрагмента изображения $3 \times 3$ пикселя. Из приведенных кривых следует, что при выборе подходящих направлений освещения и наблюдения можно зарегистрировать уменьшение $\eta$ до нуля при значениях $N$, составляющих $50 \%$ от максимального значения $N$, равного 89000 циклам. Полученные данные могут быть основой для создания в дальнейшем физических моделей многоцикловой усталости материалов и методов неразрушающей диагностики остаточного ресурса деталей техники. Предельное значение растягивающей деформации, найденное в настоящей работе, по порядку величины совпадает с предельным значением деформации, возникающей при квазистатических испытаниях на разрыв стандартных образцов. Данное совпадение позволяет предположить, что методы неразрушающего контроля, способы оценки поврежденности объектов методами механики сплошных сред и методы численного расчета напряженно-деформированного состояния тел, разработанные для квазистатических нагружений, могут быть адаптированы для изучения явлений, возникающих при многоцикловой усталости элементов конструкций.

\section{Благодарности}

Авторы благодарят И.С. Каманцева за помощь в проведении экспериментов.

\section{Финансирование работы}

Работа выполнена при частичной поддержке акта 211 Правительства РФ (соглашение № 02.А03.21.0006).

\section{Конфликт интересов}

Авторы заявляют, что у них нет конфликта интересов.

\section{Список литературы}

[1] Д.А. Тупикин, Контроль. Диагностика, № 11, 53 (2003).

[2] И.И. Новиков, В.А. Ермишин, Физическая механика реальных материалов (Наука, М., 2004).

[3] J. Lasar, M. Hola, O. Cip, in Book of abstracts. Conference PhotoMechanics (Delft University, Netherlands, 2015), p. 64.

[4] Г.Дж. Гаф, Усталость металлов (Глав. ред. лит-ры по черной металлургии, М.-Л., 1935).

[5] В.Ф. Терентьев, Усталость металлических материалов (Наука, М., 2002).
[6] Y. Murakami, Metal fatigue: effects of small defects and nonmetallic inclusions (Academic Press, 2019).

[7] S.S. Manson, Exp. Mech., 5 (7), 193 (1965).

[8] J. Schijve, Int. J. Fatigue, 25 (8), 679 (2003).

[9] Р.К. Эрф, Голографические неразрушающие исследования (Машиностроение, М., 1985).

[10] E. Marom, R.K. Muller, Int. J. Nondestructive Testing, 3, 171 (1971).

[11] В.П. Козубенко, В.А Потиченко, Ю.С. Бородин, Проблемы прочности, № 7, 103 (1989).

[12] А.П. Владимиров, И.С. Каманцев, В.Е. Веселова, Э.С. Горкунов, С.В. Гладковский, ЖТФ, 86 (4), 85 (2016).

[13] A.P. Vladimirov, Opt. Eng., 55 (12), 1217 (2016).

[14] А.П. Владимиров, И.С. Каманцев, Н.А. Друкаренко, Л.А. Акашев, А.В. Дружинин, Оптика и спектроскопия, 127 (11), 870 (2019). DOI: $10.21883 /$ OS.2019.11.48530.165-19

[15] Л.Б. Зуев, В.И. Данилов, Н.М. Мних, Завод. лаб., 56 (2), 90 (1990).

[16] M.A. Sutton, J.-J. Orteu, H. Schreier, Image correlation for shape, motion and deformation measurements (University of South Carolina, Columbia, USA, 2009).

[17] С.В. Панин, П.С. Любутин, В.В. Титков, Анализ изображений в оптическом методе оценки деформации (Издво СО РАН, Томск, 2017).

[18] A. Gilanyi, K. Morishita, T. Sukegawa, M. Uesaka, K. Miya, Fusion Eng. Design, 42 (1-4), 485 (1998).

[19] В.А. Ермишкин, Д.П. Мурат, В.В. Подбельский, Автоматизация и современные технологии, № 2, 11 (2008).

[20] О.А. Плехов, И.А. Пантелеев, В.А. Леонтьев, Физ. мезомеханика, 12 (5), 37 (2009). 